\title{
Synthesis of a hexasaccharide acceptor corresponding to the reducing terminus of mycobacterial 3-O-methylmannose polysaccharide $(\mathrm{MMP})^{1}$
}

\author{
Wensheng Liao * , Depei Lu * \\ Research Center for Eco-Environmental Sciences, Academia Sinica, P.O. Box 2871, Beijing 100085, \\ PR China
}

Received 5 June 1996; accepted in revised form 8 September 1996

\begin{abstract}
The title compound methyl $O$-(2,6-di- $O$-benzyl-3- $O$-methyl- $\alpha$-D-mannopyranosyl)-[(1 $\rightarrow 4)$ $O$-(2,6-di- $O$-benzyl-3- $O$-methyl- $\alpha$-D-mannopyranosyl) $]_{4}$-(1 $\rightarrow$ 4)-2,6-di- $O$-benzyl-3- $O$-methyl- $\alpha$-Dmannopyranoside (2) was synthesized in a blockwise manner, employing trichloroacetimidate (11) and (20) as glycosyl donors. The strategy relies on the single-step preparation of the 3-O-methyl ethers (4) and (12) as starting materials. Since all intermediates contain one or more $\mathrm{OCH}_{3}$ groups, they are easily identified by NMR spectroscopy using the methyl proton signals. Compound 2 corresponds to the reducing terminal hexasaccharide of mycobacterial 3-O-methylmannose polysaccharide (MMP). MMP has the unusual property of stimulating the fatty acid synthetase multienzyme complex. Compound 2 can serve as a suitable glycosyl acceptor for the synthesis of extended fragments of MMP. (c) 1996 Elsevier Science Ltd.
\end{abstract}

Keywords: Synthesis; Glycosylation; 3-O-Methylmannose polysaccharide (MMP); Mycobacterium

\section{Introduction}

In recent decades, mycobacteria have been demonstrated to be one of the few higher organisms that have adapted the helical-forming, lipid-binding property of amylose-like

\footnotetext{
* Corresponding authors.

${ }^{1}$ Project supported by the National Natural Science Foundation of China.
} 
oligosaccharides to regulate metabolic process [1,2]. Several polymethylpolysaccharides (PMPS) are found in Mycobacterium smegmatis [3,4], including a neutral polysaccharide (MMP) that contains 3-O-methyl-D-mannose. MMP can form a stable 1:1 complex with long-chain fatty acids and acyl coenzyme A derivatives in which the lipid is included in the interior of the helically coiled polysaccharide in a specific orientation $[5,6]$. The role of 3-O-methylation may be to stabilize the helical conformation of MMP as well as to enhance the direct interaction with lipids due to the hydrophobic nature of the methyl groups. It is postulated that MMP, along with other PMPs, serve as lipid carriers in the cell. They activate the fatty acid synthetase complex and alter the fatty acid product distribution [7].

The structure of MMP has been well defined [2,6]. It is composed of 10-13 3-O-methylmannoses joined by $\alpha-(1 \rightarrow 4)$ linkages, each molecule is terminated by an unmethylated mannose, and the reducing end of the linear chain is blocked by an $\alpha$-methyl aglycon.

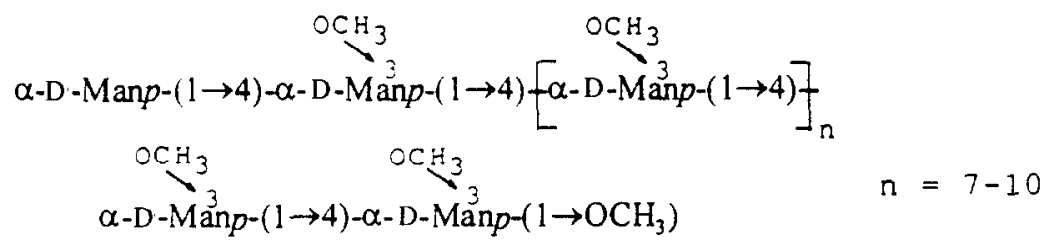

As part of a project on the total synthesis of MMP, we describe here a regio- and stereo-controlled synthesis of the hexasaccharide glycosyl acceptor 2 , which corresponds to the reducing terminal fragment of MMP. Compound $\mathbf{2}$ is suitably functionalized to serve as a precursor of higher-order structures.

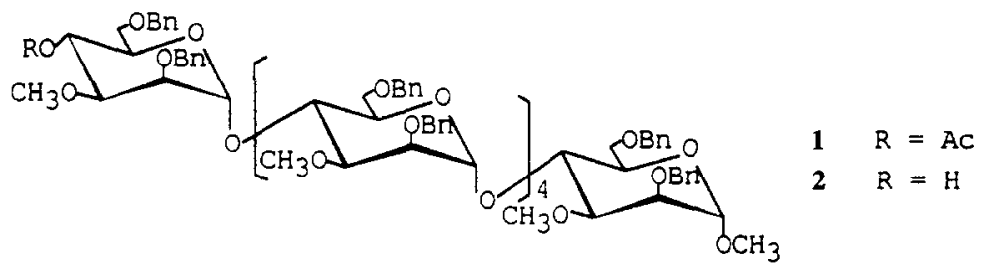

\section{Results and discussion}

Based on retrosynthetic considerations, three monosaccharide synthons $\mathbf{8 , 1 1}$, and $\mathbf{1 5}$ were designed; 11 can be obtained from 8 in a three-step sequence.

The monosaccharide units. - A method for the single-step 3-O-methylation of methyl glycopyranoside, which made large-scale preparation of the starting material 12 easy, 
has been reported [8]. The method can also be applied to allyl glycopyranosides. Thus, allyl 3-O-methyl ether 4 was obtained in this fashion in $73 \%$ yield. The ${ }^{13} \mathrm{C}$ NMR spectrum of 5 contains a deshielded signal for C-3 at 76.8 , in agreement with the 3- $O$-methyl structure. With $\mathrm{O}-3$ protected by a methyl group, benzylidenation of 4 in the presence of $\mathrm{ZnCl}_{2}$ and $\alpha, \alpha$-diethoxytoluene (instead of benzaldehyde) at room temperature gave a high yield of $6(86 \%)[9,10]$. Compound 6 was then benzylated $(\mathrm{BnBr} / \mathrm{THF})$ to give the benzylidene acetal 7 , followed by reductive ring-opening with sodium cyanoborohydride-hydrogen chloride $\left(\mathrm{NaCNBH}_{3} / \mathrm{HCl}\right)$ [11-13]. This procedure produced highly regioselective 6- $O$-benzyl ether $8(89 \%)$ containing a free $\mathrm{OH}$ group at HO-4.
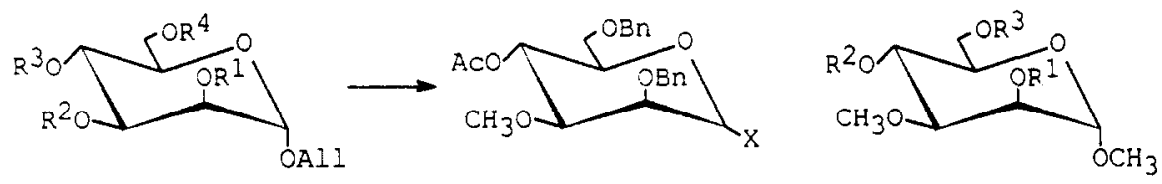

\begin{tabular}{lllll} 
& $\mathrm{R}^{1}$ & $\mathrm{R}^{2}$ & $\mathrm{R}^{3}$ & $\mathrm{R}^{4}$ \\
\cline { 2 - 5 } $\mathbf{3}$ & $\mathrm{H}$ & $\mathrm{H}$ & $\mathrm{H}$ & $\mathrm{H}$ \\
$\mathbf{4}$ & $\mathrm{H}$ & $\mathrm{CH}_{3}$ & $\mathrm{H}$ & $\mathrm{H}$ \\
$\mathbf{5}$ & $\mathrm{AC}$ & $\mathrm{CH}_{3}$ & $\mathrm{AC}$ & $\mathrm{AC}$ \\
$\mathbf{6}$ & $\mathrm{H}$ & $\mathrm{CH}_{3}$ & \multicolumn{2}{c}{$\mathrm{PhCH}$} \\
$\mathbf{7}$ & $\mathrm{Bn}$ & $\mathrm{CH}_{3}$ & \multicolumn{2}{c}{$\mathrm{PhCH}$} \\
$\mathbf{8}$ & $\mathrm{Bn}$ & $\mathrm{CH}_{3}$ & $\mathrm{H}$ & $\mathrm{Bn}$ \\
$\mathbf{9}$ & $\mathrm{Bn}$ & $\mathrm{CH}_{3}$ & $\mathrm{AC}$ & $\mathrm{Bn}$
\end{tabular}

\begin{tabular}{llll} 
& $\mathrm{R}^{2}$ & $\mathrm{R}^{2}$ & $\mathrm{R}^{3}$ \\
& $\mathrm{H}$ & $\mathrm{H}$ & $\mathrm{H}$ \\
$\mathbf{1 3}$ & $\mathrm{H}$ & \multicolumn{2}{c}{$\mathrm{PhCH}$} \\
14 & $\mathrm{Bn}$ & \multicolumn{2}{c}{$\mathrm{PhCH}$} \\
15 & $\mathrm{Bn}$ & $\mathrm{H}$ & $\mathrm{Bn}$ \\
16 & $\mathrm{Bn}$ & $\mathrm{AC}$ & $\mathrm{Bn}$ \\
17 & $\mathrm{Bn}$ & $\mathrm{Bn}$ & $\mathrm{H}$
\end{tabular}

$$
\begin{aligned}
& \mathrm{Bn}=\text { benzyl } \\
& \mathrm{Ph}=\text { phenyl }
\end{aligned}
$$
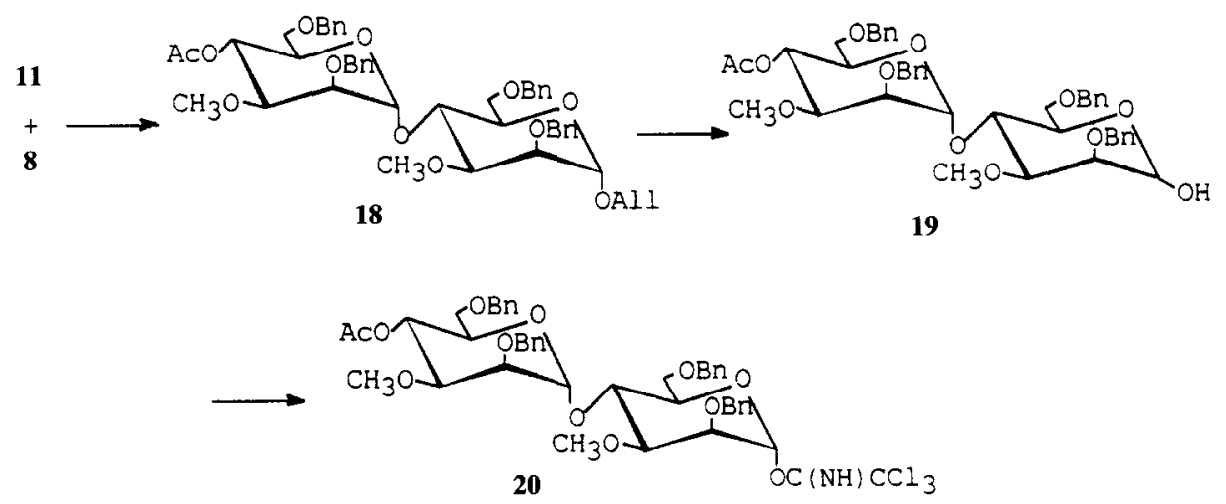

With the monosaccharide glycosyl acceptor 8 in hand, we prepared the monosaccharide glycosyl donor 11 from 8 by successive treatment with (1) acetic anhydride in pyridine by standard methods, (2) deallylation of 9 in methanol with $\mathrm{PdCl}_{2}$ as the 
catalyst $[14,15]$ to generate hemiacetal $10(87 \%)$, and (3) trichloroacetimidation of 10 with 1,8-diazabicyclo[5.4.0]undec-7-ene (DBU) and $\mathrm{CCl}_{3} \mathrm{CN}[16,17]$ in anhyd. $\mathrm{CH}_{2} \mathrm{Cl}_{2}$ $(92 \%)$.

Another monosaccharide glycosyl acceptor 15 was prepared in an analogous fashion to the synthesis of 8 . Benzylidenation of 12 afforded benzylidene acetal 13 in $83 \%$ yield, followed by benzylation to give 14. After regioselective ring-opening reduction as described for 7, the acetal 14 was converted to 15 in $89 \%$ yield; using $\mathrm{LiAlH}_{4} / \mathrm{AlCl}_{3}$ as reductant [18], the alcohol 17 was obtained with a free $\mathrm{OH}$ group at O-6.

Glycosidation reactions.-Coupling of the imidate 11 with the alcohol 8 in the presence of triethylsilyl trifluoromethanesulfonate (TESTfl) and powdered $4 \AA$ molecular sieves at $-78^{\circ} \mathrm{C}$ afforded the protected allyl mannobioside 18. The stereochemistry of the glycosidation reaction was assigned as $\alpha$-D from the ${ }^{13} \mathrm{C}$ NMR data, which included two signals for $\mathrm{C}-1_{\mathrm{A}}$ and $\mathrm{C}-1_{\mathrm{B}}$ at $\delta 96.7\left({ }^{1} J_{\mathrm{C}, \mathrm{H}} 168.6 \mathrm{~Hz}\right)$ and $99.9\left({ }^{1} J_{\mathrm{C}, \mathrm{H}}\right.$ $169.9 \mathrm{~Hz}$ ), respectively. It is worth noting that deallylation of the disaccharide 18 failed with $\mathrm{PdCl}_{2}$ in $\mathrm{MeOH}$ as described for monosaccharide $\mathbf{9}$, but proceeded well in a mixed solvent of 1:1 $\mathrm{MeOH}-\mathrm{CH}_{2} \mathrm{Cl}_{2}$ to give hemiacetal $19(87 \%)$. The desired disaccharide glycosyl imidate (20) was then obtained from 19 in $85 \%$ yield by treatment with trichloroacetonitrile in the presence of DBU.

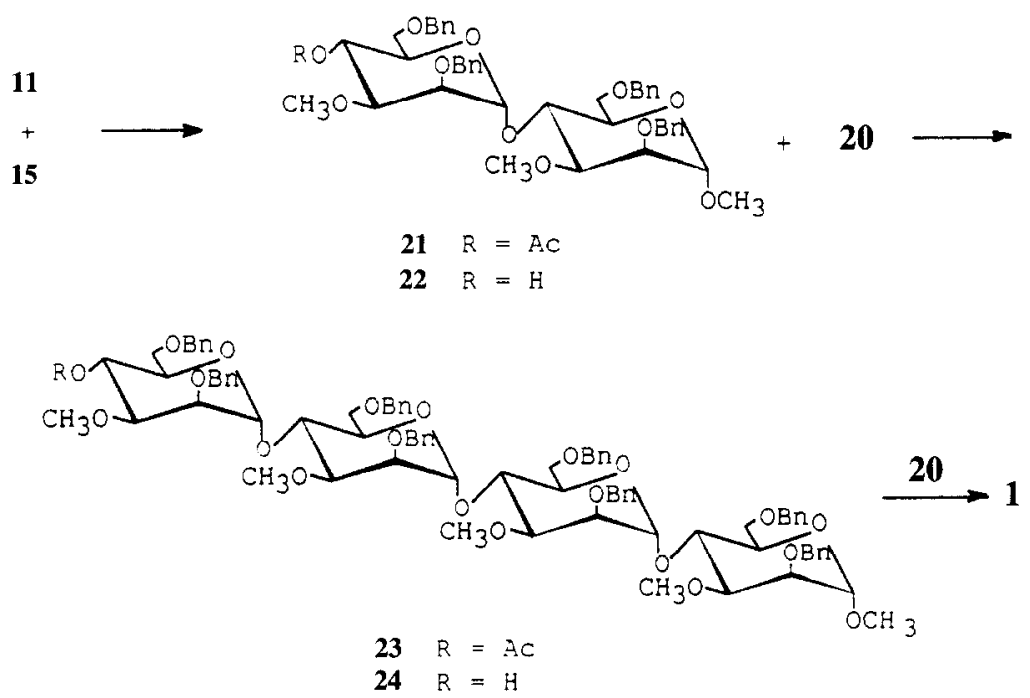

Glycosidation of the imidate 11 with the methyl glycopyranoside 15 was executed as described for 18 to provide the protected methyl mannobioside (21). Again, the reaction was performed in a highly $\alpha$-stereoselective manner. Compound 21 was deacetylated to give disaccharide glycosyl acceptor 22 . We performed blockwise chain elongation of 22 with the imidate 20 to afford the protected tetrasaccharide $(23)(61 \%)$. The structure of 23 was confirmed by ${ }^{13} \mathrm{C}$ NMR spectroscopy, in which four signals for $\mathrm{C}-1$ in the $\alpha$-D configuration appeared at $\delta 99.9\left({ }^{1} J_{\mathrm{C}, \mathrm{H}} 171.6 \mathrm{~Hz}\right), 99.8\left({ }^{1} J_{\mathrm{C}, \mathrm{H}} 170.1 \mathrm{~Hz}\right), 99.7\left({ }^{1} J_{\mathrm{C}, \mathrm{H}}\right.$ 
$169.1 \mathrm{~Hz})$, and $98.6\left({ }^{1} J_{\mathrm{C}, \mathrm{H}} 168.2 \mathrm{~Hz}\right)$. Subsequent deacetylation of 23 provided mannotetraosyl glycosyl acceptor 24. Compound 24 gave five methyl singlets at $\delta 3.37$, $3.31,3.23,3.20$, and 3.19 in its ${ }^{1} \mathrm{H}$ NMR spectrum, of which $\delta 3.37$ was assigned to the methyl aglycon (determined by NOE-DIFF technique), and the others to the 3-O-methyl groups.

The final condensation of the disaccharide donor 20 with the tetrasaccharide acceptor 24 proceeded at $-78{ }^{\circ} \mathrm{C}$ without incident. The reaction was complete in $15 \mathrm{~min}$, and produced hexasaccharide $\mathbf{1}(52 \%)$. The ${ }^{1} \mathrm{H}$ NMR spectrum of 1 showed clearly seven methyl singlets at $3.37,3.24,3.19,3.18,3.16,3.15$, and 3.10. After deacetylation, the target glycosyl mannohexaosyl acceptor 2 was obtained.

In conclusion, the linear methyl $\alpha$-(1 $\rightarrow 4)$-D-mannohexasaccharide 2 was achieved by a blockwise strategy employing disaccharide donor 20 and disaccharide acceptor 22 as building blocks. Stereochemistry of all synthetic intermediates was deduced from the synthetic sequences, and intermediates and products were characterized by their ${ }^{13} \mathrm{C}$ and ${ }^{1} \mathrm{H}$ NMR spectra. Compounds 1 and 2 were further characterized by microanalysis. Owing to their hydrophobic nature, field desorption mass spectra (FDMS) were obtained to confirm composition. In the case of 1 , signals appearing at $\mathrm{m} / z 2212$ and $\mathrm{m} / \mathrm{z} 2235$ were assigned to the $[\mathrm{M}]^{+}$and $[\mathrm{M}+\mathrm{Na}]^{+}$ions, respectively.

\section{Experimental}

General methods.-Preparative chromatography was performed on columns $(16 \times$ $240,18 \times 300$ and $35 \times 400 \mathrm{~mm})$ of silica gel (120-200 mesh). Thin-layer chromatography (TLC) was performed on silica gels $\mathrm{G}$ and $\mathrm{HF}$, and products were detected either by charring with $30 \%(\mathrm{v} / \mathrm{v}) \mathrm{H}_{2} \mathrm{SO}_{4}$ in $\mathrm{MeOH}$ or by UV light. Analytical LC was carried out in stainless-steel columns packed with silica gel $(10 \times 150 \mathrm{~mm}$, or $4.6 \times 250$ $\mathrm{mm}$ ), with peak detection by a differential refractometer (Perkin-Elmer LC-25 RI Detector), using petroleum ether-ethyl acetate as the eluent at a flow rate of 1-4 $\mathrm{mL} / \mathrm{min}$. Optical rotations were determined with a Perkin-Elmer model $241 \mathrm{MC}$ automatic polarimeter for solutions in a $1-\mathrm{dm}$, jacketed cell. Solvents were distilled before use and were dried, as necessary, by literature procedures. ${ }^{1} \mathrm{H}$ and ${ }^{13} \mathrm{C} \mathrm{NMR}$ spectra were recorded on Varian XL-400 and Varian XL-200 spectrometers; chemical shifts $(\delta)$ are given in ppm, downfield from $\mathrm{Me}_{4} \mathrm{Si}$, for solutions in $\mathrm{CDCl}_{3}$, unless noted otherwise. Subscripts A-F refer to the individual sugar residues, with A standing for the reducing-end unit. Field desorption mass spectra (FDMS) were recorded on a Finnigan MAT 90 mass spectrometer.

Allyl 3-O-methyl- $\alpha$-D-mannopyranoside (4). - A mixture of allyl $\alpha$-D-mannopyranoside 3 [19] (10.0 g, $45.5 \mathrm{mmol})$ and dibutyltin oxide $(13.5 \mathrm{~g}, 54.0 \mathrm{mmol})$ in dry $\mathrm{MeOH}$ $(250 \mathrm{~mL})$ was boiled under reflux. After the solution became transparent, heating was continucd for $1.5 \mathrm{~h}$, and the solvent was concentrated in vacuo. The resulting white foamy residue was dissolved in $N, N$-dimethylformamide $(225 \mathrm{~mL})$, and methyl iodide (7.0 mL, 2.5 molar equiv) was added. The flask was capped and heated at $80^{\circ} \mathrm{C}$ for 10 h. TLC $\left(6: 1 \mathrm{CH}_{2} \mathrm{Cl}_{2}-\mathrm{MeOH}\right)$ showed the presence of major product $\left(R_{f} 0.52\right)$ together with a small amount of starting material $\left(R_{f} 0.31\right)$. After cooling, the mixture was 
filtered. The filtrate was evaporated to a syrup, which was applied to a column of silica gel in EtOAc to give $4(7.8 \mathrm{~g}, 73 \%) ;[\alpha]_{\mathrm{D}}+58.5^{\circ}(c 0.5, \mathrm{MeOH}) ;{ }^{1} \mathrm{H}$ NMR: $\delta$ $6.12-5.86\left(\mathrm{~m}, 1 \mathrm{H}, \mathrm{CH}_{2}=\mathrm{CH}-\mathrm{CH}_{2}\right), 5.48-5.20\left(\mathrm{~m}, 2 \mathrm{H}, \mathrm{CH}_{2}=\mathrm{CH}-\mathrm{CH}_{2}\right), 4.91(\mathrm{~d}, 1$ $\left.\mathrm{H}, J_{1,2} 1.7 \mathrm{~Hz}, \mathrm{H}-1\right), 3.57\left(\mathrm{~s}, 3 \mathrm{H}, \mathrm{OCH}_{3}\right)$. Anal. Calcd for $\mathrm{C}_{10} \mathrm{H}_{18} \mathrm{O}_{6}: \mathrm{C}, 51.27 ; \mathrm{H}$, 7.74. Found: C, 51.07; H, 7.69.

The triacetate 5 was obtained by standard methods as a syrup; $[\alpha]_{\mathrm{D}}+33.9^{\circ}(c 0.8$, $\left.\mathrm{CHCl}_{3}\right) ;{ }^{13} \mathrm{C} \mathrm{NMR}: \delta 170.7,170.3,169.8\left(3 \mathrm{CH}_{3} \mathrm{CO}\right), 133.0\left(\mathrm{CH}_{2}=\mathrm{CH}-\mathrm{CH}_{2}\right), 118.3$ $\left(\mathrm{CH}_{2}=\mathrm{CH}-\mathrm{CH}_{2}\right), 96.8(\mathrm{C}-1), 76.8(\mathrm{C}-3), 68.5(\mathrm{C}-5), 67.7(\mathrm{C}-2), 67.4(\mathrm{C}-4), 62.7$ (C-6), $57.7\left(\mathrm{OCH}_{3}\right), 21.0,20.8,20.7\left(3 \mathrm{CH}_{3} \mathrm{CO}\right)$. Anal. Calcd for $\mathrm{C}_{16} \mathrm{H}_{24} \mathrm{O}_{9}: \mathrm{C}, 53.33$; $\mathrm{H}, 6.71$. Found: C, 53.25; $\mathrm{H}, 6.66$.

Allyl 4,6-O-benzylidene-3-O-methyl- $\alpha$-D-mannopyranoside (6).-Compound 4 (46.0 $\mathrm{g}, 0.20 \mathrm{~mol})$ was dissolved in $\alpha, \alpha$-diethoxytoluene $(69.0 \mathrm{~g}, 0.38 \mathrm{~mol})$. Freshly dried $\mathrm{ZnCl}_{2}(20.0 \mathrm{~g})$ was added. The mixture was stirred vigorously at room temperature for 4 h. No starting material 4 remained after this time (TLC, 3:1 petroleum ether-EtOAc). $\mathrm{CH}_{2} \mathrm{Cl}_{2}(200 \mathrm{~mL})$ was added to dilute the mixture. The precipitate was filtered off and washed with $\mathrm{CH}_{2} \mathrm{Cl}_{2}$. The filtrate and washings were combined, washed with aqueous sodium hydrogen carbonate, then water, dried $\left(\mathrm{Na}_{2} \mathrm{SO}_{4}\right)$, and concentrated to a syrup. Purification of the syrup by column chromatography $(3: 1$ petroleum ether-EtOAc) yielded $6(54.5 \mathrm{~g}, 86 \%) ;[\alpha]_{\mathrm{D}}+72^{\circ}\left(c\right.$ 1.4, $\left.\mathrm{CHCl}_{3}\right) ;{ }^{1} \mathrm{H}$ NMR: $\delta 7.54-7.30(\mathrm{~m}, 5 \mathrm{H}$, aromatic $\mathrm{H}), 6.03-5.82\left(\mathrm{~m}, 1 \mathrm{H}, \mathrm{CH}_{2}=\mathrm{CH}-\mathrm{CH}_{2}\right), 5.39-5.20\left(\mathrm{~m}, 2 \mathrm{H}, \mathrm{CH}_{2}=\mathrm{CH}-\right.$ $\left.\mathrm{CH}_{2}\right), 5.59(\mathrm{~s}, 1 \mathrm{H}, \mathrm{PhCH}), 4.94\left(\mathrm{~d}, 1 \mathrm{H}, J_{1,2} 1.3 \mathrm{~Hz}, \mathrm{H}-1\right), 4.30-3.92(\mathrm{~m}, 3 \mathrm{H}$, $\mathrm{H}-2,4,5), 3.90-3.82(\mathrm{~m}, 2 \mathrm{H}, \mathrm{H}-6), 3.71\left(\mathrm{dd}, 1 \mathrm{H}, J_{2,3} 3.4, J_{3,4} 9.3 \mathrm{~Hz}, \mathrm{H}-3\right), 3.56$ (s, 3 $\mathrm{H}, \mathrm{OC}_{3}$ ), 2.04 (br s, $\left.1 \mathrm{H}, \mathrm{OH}\right) ;{ }^{13} \mathrm{C} \mathrm{NMR:} \delta 137.3$ (aromatic C-1), $133.3\left(\mathrm{CH}_{2}=\mathrm{CH}-\right.$ $\left.\mathrm{CH}_{2}\right), 128.8,128.0,126.0$ (aromatic C), $117.7\left(\mathrm{CH}_{2}=\mathrm{CH}-\mathrm{CH}_{2}\right), 101.5(\mathrm{PhCH}), 99.1$ (C-1), 78.5 (C-3), $77.2(\mathrm{C}-4), 68.9(\mathrm{C}-2), 68.6(\mathrm{C}-6), 68.0\left(\mathrm{CH}_{2}-\mathrm{CH}=\mathrm{CH}_{2}\right), 63.2$ (C-5), $58.5\left(\mathrm{CH}_{3} \mathrm{O}\right)$. Anal. Calcd for $\mathrm{C}_{17} \mathrm{H}_{22} \mathrm{O}_{6}$ : C, 63.34; $\mathrm{H}, 6.88$. Found: $\mathrm{C}, 63.46 ; \mathrm{H}$, 6.84 .

Allyl 2-O-benzyl-4,6-O-benzylidene-3-O-methyl- $\alpha$-D-mannopyranoside (7).-To a solution of $6(37.0 \mathrm{~g}, 0.115 \mathrm{~mol})$ in dry THF $(350 \mathrm{~mL})$ cooled in an ice bath was added sodium hydride ( $80 \%$ in oil; $11.0 \mathrm{~g}, 0.37 \mathrm{~mol}$ ) with stirring. Benzyl bromide $(15.9 \mathrm{~mL}$, $0.131 \mathrm{~mol}$ ) was added dropwise and the mixture was heated under reflux with vigorous agitation for $5 \mathrm{~h}$. TLC $(3: 1$ petroleum ether-EtOAc) indicated that the reaction was complete. After cooling, the remaining sodium hydride was filtered off and the filtrate was concentrated. The residue was partitioned between water and dichloromethane. The organic layer was washed with saturated saline, dried $\left(\mathrm{Na}_{2} \mathrm{SO}_{4}\right)$, and concentrated to a syrup, which was chromatographed on a column of silica gel (4:1 petroleum etherEtOAc) to give $7(43.6 \mathrm{~g}, 92 \%) ;[\alpha]_{\mathrm{D}}+47.1^{\circ}\left(c 0.5, \mathrm{CHCl}_{3}\right) ;{ }^{1} \mathrm{H}$ NMR: $\delta$ 7.54-7.30 $(\mathrm{m}, 10 \mathrm{H}$, aromatic $\mathrm{H}), 5.97-5.76\left(\mathrm{~m}, 1 \mathrm{H}, \mathrm{CH}_{2}=\mathrm{CH}-\mathrm{CH}_{2}\right), 5.63(\mathrm{~s}, 1 \mathrm{H}, \mathrm{PhCH})$, 5.33-5.15 (m, $\left.2 \mathrm{H}, \mathrm{CH}_{2}=\mathrm{CH}-\mathrm{CH}_{2}\right), 4.86\left(\mathrm{~d}, 1 \mathrm{H}, J_{1.2} 1.7 \mathrm{~Hz}, \mathrm{H}-1\right), 4.82$ and 4.70 (ABq, $2 \mathrm{H}, J 12.4 \mathrm{~Hz}, \mathrm{PhCH}_{2}$ ), 4.29-4.11 (m, I H, H-5), 4.01-3.80 (m, $4 \mathrm{H}, \mathrm{H}-2,4,6$ and $\left.6^{\prime}\right), 3.75\left(\mathrm{dd}, 1 \mathrm{H}, J_{2,3} 3.4, J_{3,4} 10.2 \mathrm{~Hz}, \mathrm{H}-3\right), 3.51\left(\mathrm{~s}, 3 \mathrm{H}, \mathrm{OC} H_{3}\right) ;{ }^{13} \mathrm{C}$ NMR: $\delta$ 137.9 and 137.5 (aromatic C-1), $133.4\left(\mathrm{CH}_{2}=\mathrm{CH}-\mathrm{CH}_{2}\right), 128.9-126.1$ (aromatic $\mathrm{C}$ ), $117.7\left(\mathrm{CH}_{2}=\mathrm{CH}-\mathrm{CH}_{2}\right), 101.5(\mathrm{PhCH}), 98.2(\mathrm{C}-1), 78.9(\mathrm{C}-3), 77.9(\mathrm{C}-4), 75.4(\mathrm{C}-2)$, $73.4\left(\mathrm{PhCH}_{2}\right), 68.7(\mathrm{C}-6), 67.9\left(\mathrm{CH}_{2}-\mathrm{CH}=\mathrm{CH}_{2}\right), 64.1(\mathrm{C}-5), 58.6\left(\mathrm{OCH}_{3}\right)$. Anal. Calcd for $\mathrm{C}_{24} \mathrm{H}_{28} \mathrm{O}_{6}$ : C, 69.89; H, 6.84. Found: C, 70.01; H, 6.83. 
Allyl 2,6-di-O-benzyl-3-O-methyl- $\alpha$-D-mannopyranoside (8).-Hydrogen chloride in diethyl ether was added dropwise at $0{ }^{\circ} \mathrm{C}$ to compound $7(18.0 \mathrm{~g}, 43.7 \mathrm{mmol})$ and sodium cyanoborohydride $(33.0 \mathrm{~g}, 0.53 \mathrm{~mol})$ in tetrahydrofuran (distilled from lithium aluminum hydride, $300 \mathrm{~mL}$ ) containing $3 \AA$ molecular sieves until the evolution of gas ceased ( $\mathrm{pH} 5$ ). TLC (3:1 petroleum ether-EtOAc) after 15 min indicated complete reaction. The mixture was neutralized with solid sodium carbonate and filtered. The filtrate was concentrated, and the residue was partitioned between water and dichloromethane. The organic layer was dried $\left(\mathrm{Na}_{2} \mathrm{SO}_{4}\right)$ and concentrated. The resulting syrup was applied to a column of silica gel which was eluted with 2:1 petroleum ether-EtOAc to give $\left.8(16.1 \mathrm{~g}, 89 \%) ;[\alpha]_{\mathrm{D}}+7^{\circ}(c) 1.3, \mathrm{CHCl}_{3}\right) ;{ }^{1} \mathrm{H}$ NMR: $\delta$ $7.43-7.27(\mathrm{~m}, 10 \mathrm{H}$, aromatic $\mathrm{H}), 5.99-5.78\left(\mathrm{~m}, 1 \mathrm{H}, \mathrm{CH}_{2}=\mathrm{CH}-\mathrm{CH}_{2}\right), 5.33-5.13(\mathrm{~m}$, $\left.2 \mathrm{H}, \mathrm{CH}_{2}=\mathrm{CH}-\mathrm{CH}_{2}\right), 4.95\left(\mathrm{~d}, 1 \mathrm{H}, J_{1,2} 1.7 \mathrm{~Hz}, \mathrm{H}-1\right), 4.74$ and $4.67(\mathrm{ABq}, 2 \mathrm{H}, J 11.9$ $\left.\mathrm{Hz}, \mathrm{PhCH}_{2}\right), 4.63$ and $4.57\left(\mathrm{ABq}, 2 \mathrm{H}, J 11.2 \mathrm{~Hz}, \mathrm{PhCH}_{2}\right), 4.26-3.91(\mathrm{~m}, 3 \mathrm{H}$, $\left.\mathrm{CH}_{2}=\mathrm{CH}-\mathrm{CH}_{2}, \mathrm{H}-5\right), 3.85-3.73\left(\mathrm{~m}, 4 \mathrm{H}, \mathrm{H}-2,4,6\right.$ and $\left.6^{\prime}\right), 3.49\left(\mathrm{dd}, 1 \mathrm{H}, J_{2,3} 3.3, J_{3,4}\right.$ $9.3 \mathrm{~Hz}, \mathrm{H}-3$ ), $3.35\left(\mathrm{~s}, 3 \mathrm{H}, \mathrm{OCH}_{3}\right.$ ); ${ }^{13} \mathrm{C}$ NMR: $\delta$ 138.1, 138.0 (aromatic C-1), 133.6 $\left(\mathrm{CH}_{2}=\mathrm{CH}-\mathrm{CH}_{2}\right), 128.2-127.4$ (aromatic C), $117.5\left(\mathrm{CH}_{2}=\mathrm{CH}-\mathrm{CH}_{2}\right), 96.9(\mathrm{C}-1)$, $81.0(\mathrm{C}-3), 73.4,72.5\left(2 \mathrm{PhCH}_{2}\right), 72.6(\mathrm{C}-2), 71.3(\mathrm{C}-5), 70.2(\mathrm{C}-6), 67.8\left(\mathrm{CH}_{2}=\mathrm{CH}-\right.$ $\left.\mathrm{CH}_{2}\right), 67.6(\mathrm{C}-4), 57.0\left(\mathrm{OCH}_{3}\right)$. Anal. Calcd for $\mathrm{C}_{24} \mathrm{H}_{30} \mathrm{O}_{6}: \mathrm{C}, 69.55 ; \mathrm{H}, 7.29$. Found: $\mathrm{C}, 69.61 ; \mathrm{H}, 7.37$.

Allyl 4-O-acetyl-2,6-di-O-benzyl-3-O-methyl- $\alpha$-D-mannopyranoside (9).-Compound $8(8.0 \mathrm{~g}, 19.3 \mathrm{mmol})$ was acetylated by standard methods and compound 9 was obtained in a quantitative yield as a syrup; $[\alpha]_{\mathrm{D}}+15.5^{\circ}\left(c 1.1, \mathrm{CHCl}_{3}\right) ;{ }^{1} \mathrm{H}$ NMR: $\delta$ 7.69-7.27 $(\mathrm{m}, 10 \mathrm{H}$, aromatic $\mathrm{H}), 6.00-5.78\left(\mathrm{~m}, 1 \mathrm{H}, \mathrm{CH}_{2}=\mathrm{CH}-\mathrm{CH}_{2}\right), 5.38-5.13(\mathrm{~m}, 3 \mathrm{H}$, $\left.\mathrm{CH}_{2}=\mathrm{CH}-\mathrm{CH}_{2}, \mathrm{H}-4\right), 4.92\left(\mathrm{~d}, 1 \mathrm{H}, J_{1,2} 1.9 \mathrm{~Hz}, \mathrm{H}-1\right), 4.79$ and $4.68(\mathrm{ABq}, 2 \mathrm{H}, J$ $\left.11.2 \mathrm{~Hz}, \mathrm{PhCH}_{2}\right), 4.55\left(\mathrm{~s}, 2 \mathrm{H}, \mathrm{PhCH}_{2}\right), 4.37-3.76\left(\mathrm{~m}, 3 \mathrm{H}, \mathrm{CH}_{2}=\mathrm{CH}-\mathrm{CH}_{2}, \mathrm{H}-5\right)$, 3.69-3.42 (m, $3 \mathrm{H}, \mathrm{H}-2,6$ and $\left.6^{\prime}\right), 3.35$ (s, $\left.3 \mathrm{H}, \mathrm{OCH}_{3}\right), 1.98\left(\mathrm{~s}, 3 \mathrm{H}, \mathrm{CH}_{3} \mathrm{CO}\right) ;{ }^{13} \mathrm{C}$ NMR: $\delta 169.9\left(\mathrm{CH}_{3} \mathrm{CO}\right), 138.0(2$ aromatic $\mathrm{C}-1), 133.3\left(\mathrm{CH}_{2}=\mathrm{CH}-\mathrm{CH}_{2}\right), 128.2$ 127.4 (aromatic C), $117.5\left(\mathrm{CH}_{2}=\mathrm{CH}-\mathrm{CH}_{2}\right), 97.1$ (C-1), $79.0(\mathrm{C}-3), 73.3$ (C-2), 73.4, $72.7\left(2 \mathrm{PhCH}_{2}\right), 70.3(\mathrm{C}-5), 69.8(\mathrm{C}-6), 68.9(\mathrm{C}-4), 67.9\left(\mathrm{CH}_{2}=\mathrm{CH}-\mathrm{CH}_{2}\right), 57.5$ $\left(\mathrm{OCH}_{3}\right), 20.9\left(\mathrm{CH}_{3} \mathrm{CO}\right)$. Anal. Calcd for $\mathrm{C}_{26} \mathrm{H}_{32} \mathrm{O}_{6}: \mathrm{C}, 68.40 ; \mathrm{H}, 7.06$. Found: $\mathrm{C}$, 68.32; H, 7.11.

4-O-Acetyl-2,6-di-O-benzyl-3-O-methyl-D-mannopyranoside (10).-A mixture of compound $9(7.8 \mathrm{~g}, 17.2 \mathrm{mmol})$ and $\mathrm{PdCl}_{2}(0.62 \mathrm{~g}, 3.5 \mathrm{mmol})$ in dry methanol $(90 \mathrm{~mL})$ was stirred vigorously for $4 \mathrm{~h}$ at room temperature, then filtered through Celite. The filtrate was evaporated and the residue was chromatographed on a column of silica gel in 2:1 petroleum ether-EtOAc to afford 10 (mainly $\alpha$-anomer, $6.2 \mathrm{~g}, 87 \%$ ). The data are given for the anomer only: $[\alpha]_{\mathrm{D}}+3.2^{\circ}\left(c 1.2, \mathrm{CHCl}_{3}\right)$; ${ }^{1} \mathrm{H} \mathrm{NMR}: \delta 7.50-7.26(\mathrm{~m}, 10$ $\mathrm{H}$, aromatic $\mathrm{H}), 5.23\left(\mathrm{~d}, 1 \mathrm{H}, J_{1,2} 1.7 \mathrm{~Hz}, \mathrm{H}-1\right), 5.19\left(\mathrm{t}, 1 \mathrm{H}, J_{3,4} 9.8 \mathrm{~Hz}, \mathrm{H}-4\right)$, $5.38-5.13\left(\mathrm{~m}, 2 \mathrm{H}, \mathrm{CH}=\mathrm{CH}-\mathrm{CH}_{2}\right), 4.77$ and $4.66\left(\mathrm{ABq}, 2 \mathrm{H}, J 12.2 \mathrm{~Hz}, \mathrm{PhC} H_{2}\right)$, $4.52\left(\mathrm{~s}, 2 \mathrm{H}, \mathrm{PhCH} \mathrm{H}_{2}\right), 4.15-4.05(\mathrm{~m}, 1 \mathrm{H}, \mathrm{H}-5), 3.85-3.51\left(\mathrm{~m}, 3 \mathrm{H}, \mathrm{H}-2,6\right.$ and $\left.6^{\prime}\right), 3.46$ (dd, $\left.1 \mathrm{H}, J_{2,3} 3.4 \mathrm{~Hz}, J_{3,4} 10.2 \mathrm{~Hz}, \mathrm{H}-3\right), 3.34\left(\mathrm{~s}, 3 \mathrm{H}, \mathrm{OC} \mathrm{H}_{3}\right), 1.99$ (s, $3 \mathrm{H}, \mathrm{CH}_{3} \mathrm{CO}$ ); ${ }^{13} \mathrm{C}$ NMR: $\delta 169.9\left(\mathrm{CH}_{3} \mathrm{CO}\right), 137.9-137.3$ (aromatic $\left.\mathrm{C}-1\right), 128.0-127.3$ (aromatic $\mathrm{C}$ ), 92.2 (C-1), 78.3 (C-3), 73.5 (C-2), 73.0, $72.3\left(2 \mathrm{PhCH}_{2}\right), 69.8$ (C-6), 69.3 (C-5), 68.8 (C-4), $57.4\left(\mathrm{OCH}_{3}\right), 20.7\left(\mathrm{CH}_{3} \mathrm{CO}\right)$. Anal. Calcd for $\mathrm{C}_{23} \mathrm{H}_{28} \mathrm{O}_{7}: \mathrm{C}, 66.33 ; \mathrm{H}, 6.78$. Found: C, 66.25; H, 6.79. 
O-(4-O-Acetyl-2,6-di-O-benzyl-3-O-methyl- $\alpha$-D-mannopyranosyl)-trichloroacetimidate (11).- To a mixture of compound $10(3.8 \mathrm{~g}, 9.1 \mathrm{mmol})$ and trichloroacetonitrile $(1.05 \mathrm{~mL}, 10.4 \mathrm{mmol})$ in anhyd. $\mathrm{CH}_{2} \mathrm{Cl}_{2}(30 \mathrm{~mL})$ at $-10{ }^{\circ} \mathrm{C}$ was added 1,8 -diazabicyclo[5.4.0]undec-7-ene (DBU) $(0.3 \mathrm{~mL}, 2.02 \mathrm{mmol})$. The mixture was stirred at $0{ }^{\circ} \mathrm{C}$ under argon for $2 \mathrm{~h}$. The solvent was removed by evaporation and the residue was purified by column chromatography with $2: 1$ petroleum ether-EtOAc as the eluent to yield 11 as an amorphous solid $(4.7 \mathrm{~g}, 92 \%) ;[\alpha]_{\mathrm{D}}+26.2^{\circ}\left(c 1.0, \mathrm{CHCl}_{3}\right) ;{ }^{1} \mathrm{H} \mathrm{NMR} \delta$ $8.70\left(\mathrm{~s}, 1 \mathrm{H}, \mathrm{OC}(\mathrm{N} H) \mathrm{CCl}_{3}\right), 7.65-7.18(\mathrm{~m}, 10 \mathrm{H}$, aromatic $\mathrm{H}), 6.43\left(\mathrm{~d}, 1 \mathrm{H}, J_{1,2} 1.7\right.$ $\mathrm{Hz}, \mathrm{H}-1), 5.48$ (t, $\left.1 \mathrm{H}, J_{3.4} 9.7 \mathrm{~Hz}, \mathrm{H}-4\right), 4.82,4.58\left(2 \mathrm{~s}, 4 \mathrm{H}, 2 \mathrm{PhCH} \mathrm{H}_{2}\right), 3.37$ (s, $3 \mathrm{H}$, $\left.\mathrm{OC}_{3}\right), 2.05\left(\mathrm{~s}, 3 \mathrm{H}, \mathrm{CH}_{3} \mathrm{CO}\right) ;{ }^{13} \mathrm{C} \mathrm{NMR}: \delta 169.6\left(\mathrm{CH}_{3} \mathrm{CO}\right), 160.0(\mathrm{C}=\mathrm{NH}), 137.8$, 137.5 (aromatic C-1), 129.1-127.4 (aromatic C), 95.7 (C-1), 78.6 (C-3), 73.2 (C-2), 73.1, $73.0\left(2 \mathrm{PhCH}_{2}\right), 71.9(\mathrm{C}-6), 69.3(\mathrm{C}-5), 68.4(\mathrm{C}-4), 57.8\left(\mathrm{OCH}_{3}\right), 20.8\left(\mathrm{CH}_{3} \mathrm{CO}\right)$. The compound was used immediately in the coupling reaction.

Methyl 4,6-O-benzylidene-3-O-methyl- $\alpha$-D-mannopyranoside (13). - Benzylidenation of compound $12(20.0 \mathrm{~g}, 0.1 \mathrm{~mol})$ as described for 6 afforded 13 as a syrup $(24.4 \mathrm{~g}$, $83 \%) ;[\alpha]_{\mathrm{D}}+57.6^{\circ}\left(c 2.1, \mathrm{CHCl}_{3}\right)$ (lit. [20] $\left.[\alpha]_{\mathrm{D}}^{25}+73.6^{\circ}\right) ;{ }^{1} \mathrm{H}$ NMR: $\delta$ 7.54-7.29 (m, $5 \mathrm{H}$, aromatic H), $5.60(\mathrm{~s}, 1 \mathrm{H}, \mathrm{PhCH}), 4.80\left(\mathrm{~d}, 1 \mathrm{H}, J_{1,2} 1.4 \mathrm{~Hz}, \mathrm{H}-1\right), 4.32-4.24(\mathrm{~m}, 1$ $\mathrm{H}, \mathrm{H}-5), 4.11\left(\mathrm{dd}, 1 \mathrm{H}, J_{1,2} 1.4, J_{2,3} 3.4 \mathrm{~Hz}, \mathrm{H}-2\right), 4.01\left(\mathrm{t}, 1 \mathrm{H}, J_{3,4} 9.7 \mathrm{~Hz}, \mathrm{H}-4\right)$, $3.88-3.77(\mathrm{~m}, 2 \mathrm{H}, \mathrm{H}-6), 3.67\left(\mathrm{dd}, 1 \mathrm{H}, J_{2.3} 3.4, J_{3,4} 9.7 \mathrm{~Hz}, \mathrm{H}-3\right), 3.58,3.42(2 \mathrm{~s}, 6 \mathrm{H}$, $2 \mathrm{OCH}_{3}$ ), 2.06 (br s, $1 \mathrm{H}, \mathrm{OH}$ ).

Methyl 2-O-benzyl-4,6-O-benzylidene-3-O-methyl- $\alpha$-D-mannopyranoside (14).Compound 13 ( $18.5 \mathrm{~g}, 62.5 \mathrm{mmol})$ was benzylated as described for 7 to afford 14 [21] as a syrup $(22.5 \mathrm{~g}, 93 \%) ;[\alpha]_{\mathrm{D}}+34^{\circ}\left(c 1.3, \mathrm{CHCl}_{3}\right)$; ${ }^{\prime} \mathrm{H}$ NMR: $\delta 7.59-7.32(\mathrm{~m}, 10 \mathrm{H}$, aromatic $\mathrm{H}), 5.64(\mathrm{~s}, 1 \mathrm{H}, \mathrm{PhCH}), 4.84$ and $4.74\left(\mathrm{ABq}, 2 \mathrm{H}, J 12.2 \mathrm{~Hz}, \mathrm{PhCH}_{2}\right)$, $4.32-4.25$ (m, $1 \mathrm{H}, \mathrm{H}-5), 4.18$ (t, $\left.1 \mathrm{II}, J_{3.4} 9.7 \mathrm{~Hz}, \mathrm{H}-4\right), 3.94-3.79(\mathrm{~m}, 3 \mathrm{H}, \mathrm{H}-2,6$ and $\left.6^{\prime}\right), 3.74\left(\mathrm{dd}, 1 \mathrm{H}, J_{2.3} 3.3, J_{3.4} 9.7 \mathrm{~Hz}, \mathrm{H}-3\right), 3.50,3.38\left(2 \mathrm{~s}, 6 \mathrm{H}, 2 \mathrm{OCH}_{3}\right)$.

Methyl 2,6-di-O-benzyl-3-O-methyl- $\alpha$-D-mannopyranoside (15).-Reductive ringopening of the benzylidene group in $14(15.5 \mathrm{~g}, 40.2 \mathrm{mmol})$ as described for 8 afforded 15 as a syrup $(13.8 \mathrm{~g}, 89 \%) ;[\alpha]_{\mathrm{D}}-5.3^{\circ}\left(c 2.6, \mathrm{CHCl}_{3}\right) ;{ }^{1} \mathrm{H}$ NMR: $\delta 7.46-7.31(\mathrm{~m}, 10$ $\mathrm{H}$, aromatic $\mathrm{H}), 4.81\left(\mathrm{~d}, 1 \mathrm{H}, J_{1.2} 1.5 \mathrm{~Hz}, \mathrm{H}-1\right), 4.71$ and $4.67(\mathrm{ABq}, 2 \mathrm{H}, J 11.4 \mathrm{~Hz}$, $\left.\mathrm{PhC} \mathrm{H}_{2}\right), 4.64$ and $4.61\left(\mathrm{ABq}, 2 \mathrm{H}, J 11.3 \mathrm{~Hz}, \mathrm{PhCH}_{2}\right), 3.97\left(\mathrm{t}, 1 \mathrm{H}, J_{3,4} 9.5 \mathrm{~Hz}, \mathrm{H}-4\right)$, 3.37, $3.34\left(2 \mathrm{~s}, 6 \mathrm{H}, 2 \mathrm{OC}_{3}\right), 2.07$ (br s, $\left.1 \mathrm{H}, \mathrm{OH}\right) ;{ }^{13} \mathrm{C} \mathrm{NMR}: \delta$ 138.3, 138.1 (aromatic C-1), 128.3127 .5 (aromatic C), 99.0 (C-1), 81.1 (C-3), 73.5, 72.5 (2 $\left.\mathrm{PhCH}_{2}\right), 71.9,71.2(\mathrm{C}-2,5), 70.3(\mathrm{C}-6), 67.6(\mathrm{C}-4), 57.0\left(\mathrm{OCH}_{3}-3\right), 54.8\left(\mathrm{OCH}_{3}-1\right)$. Anal. Calcd for $\mathrm{C}_{22} \mathrm{H}_{28} \mathrm{O}_{6}$ : C, 68.02; H, 7.26. Found: $\mathrm{C}, 67.96 ; \mathrm{H}, 7.26$.

Methyl 4-O-acetyl-2,6-di-O-benzyl-3-O-methyl- $\alpha$-D-mannopyranoside (16).-Compound 15 (150 mg, $0.35 \mathrm{mmol})$ was acetylated by standard methods and 16 was obtained in a quantitative yield; $[\alpha]_{\mathrm{D}}+10.7^{\circ}\left(c 0.5, \mathrm{CHCl}_{3}\right) ;{ }^{1} \mathrm{H}$ NMR: $\delta$ 7.54-7.08 $(\mathrm{m}, 10 \mathrm{H}$, aromatic $\mathrm{H}), 5.25\left(\mathrm{t}, 1 \mathrm{H}, J_{3,4} 10.0 \mathrm{~Hz}, \mathrm{H}-4\right), 4.76\left(\mathrm{~d}, 1 \mathrm{H}, J_{1,2} 1.6 \mathrm{~Hz}, \mathrm{H}-1\right)$, 4.75 and $4.70\left(\mathrm{ABq}, 2 \mathrm{H}, J 12.2 \mathrm{~Hz}, \mathrm{PhCH}_{2}\right), 4.56$ and $4.54(\mathrm{ABq}, 2 \mathrm{H}, J 11.3 \mathrm{~Hz}$, $\left.\mathrm{PhCH}_{2}\right), 3.36,3.32\left(2 \mathrm{~s}, 6 \mathrm{H}, 2 \mathrm{OCH}_{3}\right), 1.96\left(\mathrm{~s}, 3 \mathrm{H}, \mathrm{CH}_{3} \mathrm{CO}\right) ;{ }^{13} \mathrm{C} \mathrm{NMR}: \delta 169.9$ $\left(\mathrm{CH}_{3} \mathrm{CO}\right.$ ), 138.2, 138.0 (aromatic $\mathrm{C}-1$ ), 128.2-127.5 (aromatic C), 99.1 (C-1), 79.1 (C-3), $73.4(\mathrm{C}-2), 73.3,72.7\left(2 \mathrm{PhCH}_{2}\right), 70.1$ (C-5), 69.9 (C-6), 68.9 (C-4), 57.7 $\left(\mathrm{OCH}_{3}-3\right), 54.7\left(\mathrm{OCH}_{3}-1\right), 20.9\left(\mathrm{CH}_{3} \mathrm{CO}\right)$. Anal. Calcd for $\mathrm{C}_{24} \mathrm{H}_{30} \mathrm{O}_{7}: \mathrm{C}, 66.96 ; \mathrm{H}$, 7.02. Found: C, 66.75; H, 6.99. 
Methyl 2,4-di-O-benzyl-3-O-methyl- $\alpha$-D-mannopyranoside (17).-To a boiling mixture of 14 (200 mg, $0.52 \mathrm{mmol}$ ), anhyd. $\mathrm{CH}_{2} \mathrm{Cl}_{2} /$ ether $(1.5 \mathrm{~mL}, 1 / 1, \mathrm{v} / \mathrm{v})$ and $\mathrm{LiAlH}_{4}$ (30 mg) was added a solution of $\mathrm{AlCl}_{3}(105 \mathrm{mg})$ in ether, and boiling was continued for $2 \mathrm{~h}$. After cooling, EtOAc and water were added, the organic layer was separated and the aqueous layer extracted with $\mathrm{CH}_{2} \mathrm{Cl}_{2}$. The combined extracts were washed with water, dried, and evaporated. The residue was applied to a column of silica gel (petroleum ether-EtOAc) to yield $17(176 \mathrm{mg}, 87 \%) ;[\alpha]_{\mathrm{D}}+18.4^{\circ}\left(\mathrm{c} 0.5, \mathrm{CHCl}_{3}\right) ;{ }^{13} \mathrm{C}$ NMR: $\delta$ 138.4, 138.1 (aromatic C-1), 128.2-127.4 (aromatic C), 99.0 (C-1), 81.8 (C-3), 74.9, 74.6 (2 PhCH2), 73.7 (C-4), 72.4, 71.7 (C-2,5), 62.3 (C-6), $57.5\left(\mathrm{OCH}_{3}-3\right), 54.6$ $\left(\mathrm{OCH}_{3}-1\right)$. Anal. Calcd for $\mathrm{C}_{22} \mathrm{H}_{28} \mathrm{O}_{6}$ : C, 68.02; $\mathrm{H}, 7.26$. Found: $\mathrm{C}, 67.90 ; \mathrm{H}, 7.21$.

Allyl O-(4-O-acetyl-2,6-di-O-benzyl-3-O-methyl- $\alpha$-D-mannopyranosyl)-( $1 \rightarrow 4$ )-2,6di-O-benzyl-3-O-methyl- $\alpha$-D-mannopyranoside (18). - A mixture of compound 11 (3.75 $\mathrm{g}, 6.69 \mathrm{mmol})$ and $8(2.33 \mathrm{~g}, 5.63 \mathrm{mmol})$ in anhyd. $\mathrm{CH}_{2} \mathrm{Cl}_{2}(30 \mathrm{~mL})$ under argon was stirred with finely powdered $4 \AA$ molecular sieves, for $30 \mathrm{~min}$ at room temperature. The mixture was cooled to $-78{ }^{\circ} \mathrm{C}$ and triethylsilyl trifluoromethanesulfonate (TESTfl) (50 $\mu \mathrm{L}, 0.13 \mathrm{mmol}$ ) was added dropwise. The mixture was stirred for $15 \mathrm{~min}$ after which TLC (2:1 petroleum ether-EtOAc) indicated that the reaction was complete. The solution was neutralized with $\mathrm{Et}_{3} \mathrm{~N}(0.5 \mathrm{~mL})$, filtered through Celite and the filtrate was evaporated in vacuo. The residue was purified by column chromatography with $2: 1$ petroleum ether-EtOAc as the eluent to yield 18 as an amorphous solid $(3.48 \mathrm{~g}, 76 \%)$; $[\alpha]_{\mathrm{D}}+28.7^{\circ}\left(c 2.8, \mathrm{CHCl}_{3}\right){ }^{1} \mathrm{H}$ NMR: $\delta 7.49-7.05(\mathrm{~m}, 20 \mathrm{H}$, aromatic $\mathrm{H}), 6.02-5.78$ $\left(\mathrm{m}, 1 \mathrm{H}, \mathrm{CH}_{2}=\mathrm{CH}-\mathrm{CH}_{2}\right), 5.37-5.12\left(\mathrm{~m}, 2 \mathrm{H}, \mathrm{CH}_{2}=\mathrm{CH}-\mathrm{CH}_{2}\right), 5.29\left(\mathrm{t}, 1 \mathrm{H}, J_{3,4} 9.6\right.$ $\left.\mathrm{Hz}, \mathrm{H}-4_{\mathrm{B}}\right), 5.31\left(\mathrm{~d}, 1 \mathrm{H}, J_{1,2} 1.7 \mathrm{~Hz}, \mathrm{H}-1_{\mathrm{B}}\right), 4.94\left(\mathrm{~d}, 1 \mathrm{H}, J_{1,2} 1.7 \mathrm{~Hz}, \mathrm{H}-\mathrm{I}_{\mathrm{A}}\right)$, 4.73-4.44 (m, 8 H, $\left.4 \mathrm{PhCH}_{2}\right), 3.34,3.20\left(2 \mathrm{~s}, 6 \mathrm{H}, \mathrm{OCH}_{3}-3_{\mathrm{A}}, 3_{\mathrm{B}}\right), 1.97(\mathrm{~s}, 3 \mathrm{H}$, $\left.\mathrm{CH}_{3} \mathrm{CO}\right) ;{ }^{13} \mathrm{C}$ NMR: $\delta \quad 170.1 \quad\left(\mathrm{CH}_{3} \mathrm{CO}\right), 138.6-138.1$ (aromatic $\left.\mathrm{C}-1\right), 133.7$ $\left(\mathrm{CH}_{2}=\mathrm{CH}-\mathrm{CH}_{2}\right), 128.9-127.2\left(\right.$ aromatic C), $117.6\left(\mathrm{CH}_{2}=\mathrm{CH}-\mathrm{CH}_{2}\right), 99.9\left({ }^{1} J_{\mathrm{C}-1, \mathrm{H}-1}\right.$ $\left.169.9 \mathrm{~Hz}, \mathrm{C}-1_{\mathrm{B}}\right), 96.7\left({ }^{1} J_{\mathrm{C}-1, \mathrm{H}-1} 168.6 \mathrm{~Hz}, \mathrm{C}-1_{\mathrm{A}}\right), 81.7,78.7\left(\mathrm{C}-3_{\mathrm{B}}, 3_{\mathrm{A}}\right), 57.6,56.6$ $\left(\mathrm{OCH}_{3}-3_{\mathrm{B}}, 3_{\mathrm{A}}\right), 21.0\left(\mathrm{CH}_{3} \mathrm{CO}\right)$. Anal. Calcd for $\mathrm{C}_{47} \mathrm{H}_{56} \mathrm{O}_{12}: \mathrm{C}, 69.44 ; \mathrm{H}, 6.94$. Found: C, $69.78 ; \mathrm{H}, 6.94$.

O-(4-O-Acetyl-2,6-di-O-benzyl-3-O-methyl- $\alpha$-D-mannopyranosyl)- $(1 \rightarrow 4)-2,6$-di-Obenzyl-3-O-methyl-D-mannopyranoside (19).-A mixture of $18(1.3 \mathrm{~g}, 1.6 \mathrm{mmol})$ and $\mathrm{PdCl}_{2}(100 \mathrm{mg}, 0.57 \mathrm{mmol})$ in $1: 1 \mathrm{MeOH}-\mathrm{CH}_{2} \mathrm{Cl}_{2}(22.5 \mathrm{~mL})$ was stirred for $4 \mathrm{~h}$ at room temperature and filtered through Celite. The filtrate was evaporated and the residue was purified by column chromatography with 1:1 petroleum ether-EtOAc as the eluent to give 19 (mainly $\alpha$-anomer, $1.08 \mathrm{~g}, 87 \%$ ); The data are given for $\alpha$-anomer only: $[\alpha]_{D}+16.1^{\circ}\left(c\right.$ 2.6, $\left.\mathrm{CHCl}_{3}\right) ;{ }^{1} \mathrm{H}$ NMR: $\delta 7.60-7.20(\mathrm{~m}, 20 \mathrm{H}$, aromatic $\mathrm{H}), 5.33$ $\left(\mathrm{d}, 1 \mathrm{H}, J_{1,2} 1.3 \mathrm{~Hz}, \mathrm{H}-1_{\mathrm{B}}\right), 5.32\left(\mathrm{t}, 1 \mathrm{H}, J_{3,4} 9.7 \mathrm{~Hz}, \mathrm{H}-4_{\mathrm{B}}\right), 5.29\left(\mathrm{~d}, 1 \mathrm{H}, J_{1,2} 1.9 \mathrm{~Hz}\right.$, $\left.\mathrm{H}-\mathrm{I}_{\Lambda}\right), 4.79-4.41\left(\mathrm{~m}, 8 \mathrm{H}, 4 \mathrm{PhCH}_{2}\right), 4.01\left(\mathrm{t}, 1 \mathrm{H}, J_{3,4} 9.4 \mathrm{~Hz}, \mathrm{H}-4_{\mathrm{A}}\right), 3.35,3.19(2 \mathrm{~s}$, $\left.6 \mathrm{H}, \mathrm{OC}_{3}-3_{\mathrm{A}}, 3_{\mathrm{B}}\right), 2.02\left(\mathrm{~s}, 3 \mathrm{H}, \mathrm{CH}_{3} \mathrm{CO}\right) ;{ }^{13} \mathrm{C} \mathrm{NMR}: \delta 169.8\left(\mathrm{CH}_{3} \mathrm{CO}\right), 138.3-138.1$ (aromatic C-1), 128.3-127.4 (aromatic C), $100.0\left(\mathrm{C}-1_{\mathrm{B}}\right), 92.3\left(\mathrm{C}-1_{\mathrm{A}}\right), 81.2,78.7$ $\left(\mathrm{C}-3_{\mathrm{B}}, 3_{\mathrm{A}}\right)$, 57.6, $56.6\left(\mathrm{OCH}_{3}-3_{\mathrm{B}}, 3_{\mathrm{A}}\right), 20.5\left(\mathrm{CH}_{3} \mathrm{CO}\right)$. Anal. Calcd for $\mathrm{C}_{44} \mathrm{H}_{52} \mathrm{O}_{12}: \mathrm{C}$, 67.38; H, 6.78. Found: C, 67.39; H, 6.80.

$\mathrm{O}-(4-\mathrm{O}-$ Acetyl-2,6-di-O-benzyl-3-O-methyl- $\alpha$-D-mannopyranosyl)-( $1 \rightarrow 4)-2,6$-di-Obenzyl-3-O-methyl- $\alpha$-D-mannopyranosyl trichloroacetimidate (20). - To a mixture of compound $19(0.8 \mathrm{~g}, 1.04 \mathrm{mmol})$ and $\mathrm{Cl}_{3} \mathrm{CCN}(0.3 \mathrm{~mL}, 2.98 \mathrm{mmol})$ in anhyd. $\mathrm{CH}_{2} \mathrm{Cl}_{2}$ 
(15 mL) at $-10{ }^{\circ} \mathrm{C}$ was added 1,8-diazabicyclo[5.4.0]undec-7-ene (DBU) (70 $\mu \mathrm{L}, 0.46$ mmol). The mixture was stirred at $0{ }^{\circ} \mathrm{C}$ under argon for $3 \mathrm{~h}$. Processing and chromatography on silica gel in 2:1 petroleum ether-EtOAc as eluent yielded $20(810 \mathrm{mg}, 85 \%)$; $[\alpha]_{\mathrm{D}}+24.8^{\circ}\left(\mathrm{c} 0.7, \mathrm{CHCl}_{3}\right){ }^{1}{ }^{\mathrm{H}} \mathrm{NMR}: \delta 8.63\left(\mathrm{~s}, 1 \mathrm{H}, \mathrm{OC}(\mathrm{N} H) \mathrm{CCl}_{3}\right), 7.63-7.08(\mathrm{~m}$, $20 \mathrm{H}$, aromatic $\mathrm{H}), 6.44\left(\mathrm{~d}, 1 \mathrm{H}, J_{1,2} 1.7 \mathrm{~Hz}, \mathrm{H}-1_{\mathrm{A}}\right), 5.35\left(\mathrm{t}, 1 \mathrm{H}, J_{3,4} 9.3 \mathrm{~Hz}, \mathrm{H}-4_{\mathrm{B}}\right)$, $5.22\left(\mathrm{~d}, 1 \mathrm{H}, J_{1,2} 1.4 \mathrm{~Hz}, \mathrm{H}-1_{\mathrm{B}}\right), 3.34,3.16\left(2 \mathrm{~s}, 6 \mathrm{H}, \mathrm{OCH}_{3}-3_{\mathrm{A}}, 3_{\mathrm{B}}\right), 1.96(\mathrm{~s}, 3 \mathrm{H}$, $\left.\mathrm{CH}_{3} \mathrm{CO}\right) ;{ }^{13} \mathrm{C}$ NMR: $\delta 169.7\left(\mathrm{CH}_{3} \mathrm{CO}\right), 160.1(\mathrm{C}=\mathrm{NH}), 138.2-137.4$ (aromatic $\left.\mathrm{C}-1\right)$, 128.6-127.1 (aromatic C), 99.6 (C-1 $\left.{ }_{B}\right), 95.5\left(C-1_{A}\right), 81.1,78.5\left(C-3_{B}, 3_{A}\right), 57.5,56.5$ $\left(\mathrm{OCH}_{3}-3_{\mathrm{B}}, 3_{\mathrm{A}}\right), 20.8\left(\mathrm{CH}_{3} \mathrm{CO}\right)$. The compound was used immediately in the coupling reaction.

Methyl O-(4-O-acetyl-2,6-di-O-benzyl-3-O-methyl- $\alpha$-D-mannopyranosyl)-( $1 \rightarrow 4)$ 2,6-di-O-benzyl-3-O-methyl- $\alpha$-D-mannopyranoside (21). - Coupling of the imidate 11 $(1.70 \mathrm{~g}, 4.38 \mathrm{mmol})$ with the alcohol $15(2.82 \mathrm{~g}, 5.04 \mathrm{mmol})$ was performed as described for 18 to provide 21 as a syrup $(2.6 \mathrm{~g}, 76 \%) ;[\alpha]_{\mathrm{D}}+20.2^{\circ}\left(c 1.3, \mathrm{CHCl}_{3}\right) ;{ }^{1} \mathrm{H}$ NMR: $\delta 7.45-7.18(\mathrm{~m}, 20 \mathrm{H}$, aromatic $\mathrm{H}), 5.31\left(\mathrm{~d}, 1 \mathrm{H}, J_{1.2} 1.7 \mathrm{~Hz}, \mathrm{H}-1_{\mathrm{B}}\right), 5.29(\mathrm{t}, 1$ $\left.\mathrm{H}, J_{3,4} 9.8 \mathrm{~Hz}, \mathrm{H}-4_{\mathrm{B}}\right), 4.78\left(\mathrm{~d}, 1 \mathrm{H}, J_{1,2} 1.7 \mathrm{~Hz}, \mathrm{H}-1_{\mathrm{A}}\right), 4.74-4.42\left(\mathrm{~m}, 8 \mathrm{H}, 4 \mathrm{PhCH}{ }_{2}\right)$, 3.38, 3.33, $3.18\left(3 \mathrm{~s}, 9 \mathrm{H}, \mathrm{OCH}_{3}-1_{\mathrm{A}}, 3_{\mathrm{A}}, 3_{\mathrm{B}}\right), 2.19\left(\mathrm{~s}, 3 \mathrm{H}, \mathrm{CH}_{3} \mathrm{CO}\right) ;{ }^{13} \mathrm{C} \mathrm{NMR}: \delta 169.8$ $\left(\mathrm{CH}_{3} \mathrm{CO}\right.$ ), 138.4-137.9 (aromatic C-1), 128.2-127.1 (aromatic C), $99.7\left({ }^{1} J_{\mathrm{C}-1, \mathrm{H}-1} 170.3\right.$ $\left.\mathrm{Hz}, \mathrm{C}-1_{\mathrm{B}}\right), 98.5\left({ }^{1} J_{\mathrm{C}-1, \mathrm{H}-1} 169.1 \mathrm{~Hz}, \mathrm{C}-1_{\mathrm{A}}\right), 81.6,78.6\left(\mathrm{C}-3_{\mathrm{B}}, 3_{\mathrm{A}}\right), 57.4,56.4\left(\mathrm{OCH}_{3}-\right.$ $\left.3_{\mathrm{B}}, 3_{\mathrm{A}}\right), 54.7\left(\mathrm{OCH}_{3}-1_{\mathrm{A}}\right), 20.8\left(\mathrm{CH}_{3} \mathrm{CO}\right)$. Anal. Calcd for $\mathrm{C}_{45} \mathrm{H}_{54} \mathrm{O}_{12}: \mathrm{C}, 68.69 ; \mathrm{H}$, 6.92. Found: C, $68.35 ; \mathrm{H}, 6.89$.

Methyl O-(2,6-di-O-benzyl-3-O-methyl- $\alpha$-D-mannopyranosyl)-( $1 \rightarrow 4$ )-2,6-di-Obenzyl-3-O-methyl- $\alpha$-D-mannopyranoside (22). - A solution of $21(2.1 \mathrm{~g}, 2.67 \mathrm{mmol})$ in THF $(15 \mathrm{~mL})$ and $0.15 \mathrm{~N} \mathrm{NaOMe}-\mathrm{MeOH}(20 \mathrm{~mL})$ was stirred for $4 \mathrm{~h}$ at room tempcrature. The reaction mixture was ncutralized with Amberlite IR-120, filtered, and concentrated. Chromatography on silica gel in 1:1 petroleum ether-EtOAc afforded 22 $(1.88 \mathrm{~g}, 95 \%) ;[\alpha]_{\mathrm{D}}+7.4\left(c 1.7, \mathrm{CHCl}_{3}\right) ;{ }^{1} \mathrm{H}$ NMR: $\delta 7.64-7.13(\mathrm{~m}, 20 \mathrm{H}$, aromatic H), $5.34\left(\mathrm{~d}, 1 \mathrm{H}, J_{1,2} 1.6 \mathrm{~Hz}, \mathrm{H}-1_{\mathrm{B}}\right), 4.79\left(\mathrm{~d}, 1 \mathrm{H}, J_{1,2} 1.6 \mathrm{~Hz}, \mathrm{H}-1_{\mathrm{A}}\right), 4.76-4.44(\mathrm{~m}, 8$ $\mathrm{H}, 4 \mathrm{PhCH}_{2}$ ), $3.98\left(\mathrm{t}, 1 \mathrm{H}, J_{3,4} 9.7 \mathrm{~Hz}, \mathrm{H}-4_{\mathrm{B}}\right), 3.97\left(\mathrm{t}, 1 \mathrm{H}, J_{3,4} 9.7 \mathrm{~Hz}, \mathrm{H}-4_{\mathrm{A}}\right), 3.47$ (dd, $1 \mathrm{H}, J_{2,3} 3.3 \mathrm{~Hz}, J_{3,4} 9.7 \mathrm{~Hz}, \mathrm{H}-3_{\mathrm{B}}$ ), 3.38 (dd, $1 \mathrm{H}, J_{2,3} 3.4 \mathrm{~Hz}, J_{3,4} 9.8 \mathrm{~Hz}$, $\mathrm{H}-3_{\mathrm{A}}$ ), 3.36, 3.33, $3.23\left(3 \mathrm{~s}, 9 \mathrm{H}, \mathrm{OC}_{3}-1_{\mathrm{A}}, 3_{\mathrm{A}}, 3_{\mathrm{B}}\right) ;{ }^{13} \mathrm{C}$ NMR: $\delta$ 138.4, 138.2, 138.0, 137.9 (aromatic C-1), 128.2-127.1 (aromatic C), $99.6\left(\mathrm{C}-1_{\mathrm{B}}\right), 98.4\left(\mathrm{C}-1_{\mathrm{A}}\right), 81.7,80.5$ $\left(\mathrm{C}-3_{\mathrm{B}}, 3_{\mathrm{A}}\right), 56.8,56.5\left(\mathrm{OCH}_{3}-3_{\mathrm{B}}, 3_{\mathrm{A}}\right), 54.8\left(\mathrm{OCH}_{3}-\mathrm{I}_{\mathrm{A}}\right)$. Anal. Calcd for $\mathrm{C}_{43} \mathrm{H}_{52} \mathrm{O}_{11}$ : C, 69.34; H, 7.04. Found: C, 69.01; H, 6.95.

Methyl O-(4-O-acetyl-2,6-di-O-benzyl-3-O-methyl- $\alpha$-D-mannopyranosyl)-I( $1 \rightarrow 4)$ O-(2,6-di-O-benzyl-3-O-methyl- $\alpha$-D-mannopyranosyl) $]_{2}-(1 \rightarrow 4)-2,6$-di-O-benzyl-3-Omethyl- $\alpha$-D-mannopyranoside (23). - Coupling of the disaccharide donor 20 (0.96 g, $1.05 \mathrm{mmol})$ with the acceptor $22(0.52 \mathrm{~g}, 0.70 \mathrm{mmol})$ was carried out using a catalytic amount of TESTfl $(50 \mu \mathrm{L}, 0.13 \mathrm{mmol})$ as described for 18, to yield 23 as an amorphous solid $(0.45 \mathrm{~g}, 61 \%) ;[\alpha]_{\mathrm{D}}+20.9^{\circ}\left(c 1.5, \mathrm{CHCl}_{3}\right) ;{ }^{1} \mathrm{H}$ NMR: $\delta 7.70-6.99(\mathrm{~m}, 40 \mathrm{H}$, aromatic H), 5.38-5.24 (m, $\left.4 \mathrm{H}, \mathrm{H}-1_{\mathrm{D}}, \mathrm{l}_{\mathrm{C}}, 1_{\mathrm{B}}, \mathrm{H}-4_{\mathrm{D}}\right), 4.80\left(\mathrm{~d}, 1 \mathrm{H}, J_{1,2} 1.6 \mathrm{~Hz}, \mathrm{H}-1_{\mathrm{A}}\right)$, $4.75-4.37\left(\mathrm{~m}, 16 \mathrm{H}, 8 \mathrm{PhCH}_{2}\right), 3.36\left(\mathrm{~s}, 3 \mathrm{H}, \mathrm{OCH}_{3}-1_{\mathrm{A}}\right), 3.30,3.20(2 \mathrm{~s}, 6 \mathrm{H}$, $\left.\mathrm{OC}_{3}-3_{\mathrm{A}}, 3_{\mathrm{D}}\right), 3.21\left(\mathrm{~s}, 6 \mathrm{H}, \mathrm{OC}_{3}-3_{\mathrm{B}}, 3_{\mathrm{C}}\right), 2.00\left(\mathrm{~s}, 3 \mathrm{H}, \mathrm{CH}_{3} \mathrm{CO}\right) ;{ }^{13} \mathrm{C} \mathrm{NMR:} 169.9$ $\left(\mathrm{CH}_{3} \mathrm{CO}\right.$ ), 138.7 138.1 (aromatic C-1), 128.4-127.2 (aromatic C), 99.9 ( ${ }^{1} J_{\mathrm{C}, \mathrm{H}} 171.6$ $\left.\mathrm{Hz}, \mathrm{C}-1_{\mathrm{D}}\right), 99.8\left({ }^{1} J_{\mathrm{C}, \mathrm{H}} 170.1 \mathrm{~Hz}, \mathrm{C}-1_{\mathrm{C}}\right), 99.7\left({ }^{1} J_{\mathrm{C}, \mathrm{H}} 169.1 \mathrm{~Hz}, \mathrm{C}-1_{\mathrm{B}}\right), 98.6\left({ }^{1} J_{\mathrm{C}, \mathrm{H}} 168.2\right.$ 
$\left.\mathrm{Hz}, \mathrm{C}-1_{\mathrm{A}}\right), 81.7,81.4,81.1\left(\mathrm{C}-3_{\mathrm{D}}, 3_{\mathrm{C}}, 3_{\mathrm{B}}\right), 78.8\left(\mathrm{C}-3_{\mathrm{A}}\right), 57.6,56.6\left(\mathrm{OCH}_{3}-3_{\mathrm{D}}, 3_{\mathrm{C}}\right), 56.4$ $\left(\mathrm{OCH}_{3}-3_{\mathrm{B}}, 3_{\mathrm{A}}\right), 54.9\left(\mathrm{OCH}_{3}-\mathrm{I}_{\mathrm{A}}\right), 21.0\left(\mathrm{CH}_{3} \mathrm{CO}\right)$. Anal. Calcd for $\mathrm{C}_{87} \mathrm{H}_{102} \mathrm{O}_{22}: \mathrm{C}$, 69.68; H, 6.85. Found: C, 69.35; H, 6.80.

Methyl O-(2,6-di-O-benzyl-3-O-methyl- $\alpha$-D-mannopyranosyl)-l(1 $\rightarrow$ 4)-O-(2,6-di-Obenzyl-3-O-methyl- $\alpha$-D-mannopyranosyl) $]_{2}-(1 \rightarrow 4)$-2,6-di-O-benzyl-3-O-methyl- $\alpha$-Dmannopyranoside (24). - A solution of $23(0.3 \mathrm{~g}, 0.2 \mathrm{mmol})$ in THF ( $4 \mathrm{~mL})$ and $0.10 \mathrm{~N}$ $\mathrm{NaOMe}-\mathrm{MeOH}(6 \mathrm{~mL})$ was stirred for $2 \mathrm{~h}$ at room temperature. Processing and chromatography on silica gel in 3:2 petroleum ether-EtOAc gave $24(262 \mathrm{mg}, 90 \%)$; $[\alpha]_{\mathrm{D}}+14.5^{\circ}\left(c 0.8, \mathrm{CHCl}_{3}\right) ;{ }^{1} \mathrm{H}$ NMR: $\delta 7.54-7.06(\mathrm{~m}, 40 \mathrm{H}$, aromatic $\mathrm{H}), 5.38(\mathrm{~d}, 1$ $\left.\mathrm{H}, J_{1,2} 1.9 \mathrm{~Hz}, \mathrm{H}-1_{\mathrm{D}}\right), 5.29\left(\mathrm{~d}, 1 \mathrm{H}, J_{1,2} 1.8 \mathrm{~Hz}, \mathrm{H}-1_{\mathrm{C}}\right), 5.27\left(\mathrm{~d}, 1 \mathrm{H}, J_{1,2} 1.8 \mathrm{~Hz}\right.$, $\left.\mathrm{H}-1_{\mathrm{B}}\right), 4.78\left(\mathrm{~d}, 1 \mathrm{H}, J_{1,2} 1.7 \mathrm{~Hz}, \mathrm{H}-1_{\mathrm{A}}\right), 4.77-4.37\left(\mathrm{~m}, 16 \mathrm{H}, 8 \mathrm{PhC} H_{2}\right), 3.37$ (s, $3 \mathrm{H}$, $\left.\mathrm{OC}_{3}-\mathrm{l}_{\mathrm{A}}\right), 3.31,3.23,3.20,3.19\left(4 \mathrm{~s}, 12 \mathrm{H}, \mathrm{OC}_{3}-3_{\mathrm{A}}, 3_{\mathrm{B}}, 3_{\mathrm{C}}, 3_{\mathrm{D}}\right) ;{ }^{13} \mathrm{C}$ NMR: $\delta$ 138.7-138.4 (aromatic C-1), 128.4-127.2 (aromatic C), 99.8, 99.7, $99.6\left(\mathrm{C}-1_{\mathrm{D}}, 1_{\mathrm{C}}, 1_{\mathrm{B}}\right.$ ), $98.6\left(\mathrm{C}-1_{A}\right), 81.7,81.5,81.4\left(\mathrm{C}-3_{D}, 3_{C}, 3_{B}\right), 80.6\left(\mathrm{C}-3_{A}\right), 57.0,56.7\left(\mathrm{OCH}_{3}-3_{D}, 3_{C}\right), 56.4$ $\left(\mathrm{OCH}_{3}-3_{\mathrm{B}}, 3_{\mathrm{A}}\right), 54.9\left(\mathrm{OCH}_{3}-\mathrm{l}_{\mathrm{A}}\right)$. Anal. Calcd for $\mathrm{C}_{85} \mathrm{H}_{100} \mathrm{O}_{21}: \mathrm{C}, 75.24 ; \mathrm{H}, 7.43$. Found: C, 74.98; H, 7.35.

Methyl O-(4-O-acetyl-2,6-di-O-benzyl-3-O-methyl- $\alpha$-D-mannopyranosyl)-l( $1 \rightarrow 4)$ O-(2,6-di-O-benzyl-3-O-methyl- $\alpha$-D-mannopyranosyl) $]_{4}-(I \rightarrow 4)-2,6$-di-O-benzyl-3-Omethyl- $\alpha$-D-mannopyranoside (1). - Glycosylation of the imidate 20 (236 $\mathrm{mg}, 0.26$ mmol) with the tetrasaccharide acceptor $24(150 \mathrm{mg}, 0.10 \mathrm{mmol})$ was carried out using a catalytic amount of TESTfl $(15 \mu \mathrm{L}, 39 \mu \mathrm{mol})$ as described for 18, to yield 1 as an amorphous solid $(117 \mathrm{mg}, 52 \%) ;[\alpha]_{\mathrm{D}}+26.6^{\circ}\left(c 0.9, \mathrm{CHCl}_{3}\right) ;{ }^{1} \mathrm{H} \mathrm{NMR}: \delta$ 7.80-7.63 $(\mathrm{m}, 60 \mathrm{H}$, aromatic $\mathrm{H}), 5.43-5.23\left(\mathrm{~m}, 6 \mathrm{H}, \mathrm{H}-1_{\mathrm{F}}, 1_{\mathrm{E}}, 1_{\mathrm{D}}, 1_{\mathrm{C}}, 1_{\mathrm{B}}, \mathrm{H}-4_{\mathrm{F}}\right), 4.78\left(\mathrm{~d}, 1 \mathrm{H}, J_{1.2}\right.$ $\left.1.8 \mathrm{~Hz}, \mathrm{H}-1_{\mathrm{A}}\right), 4.75-4.32\left(\mathrm{~m}, 24 \mathrm{H}, 12 \mathrm{PhCH}_{2}\right), 3.36\left(\mathrm{~s}, 3 \mathrm{H}, \mathrm{OC}_{3}-1_{\mathrm{A}}\right), 3.24,3.19$, $3.18,3.17,3.16,3.10\left(6 \mathrm{~s}, 18 \mathrm{H}, \mathrm{OCH}_{3}-3_{\mathrm{A}}, 3_{\mathrm{B}}, 3_{\mathrm{C}}, 3_{\mathrm{D}}, 3_{\mathrm{E}}, 3_{\mathrm{F}}\right), 1.98\left(\mathrm{~s}, 3 \mathrm{H}, \mathrm{CH}_{3} \mathrm{CO}\right) ;{ }^{13} \mathrm{C}$ NMR: $\delta 170.0\left(\mathrm{CH}_{3} \mathrm{CO}\right), 138.7-138.2$ (aromatic $\mathrm{C}-1$ ), 128.4-127.4 (aromatic $\mathrm{C}$ ), 99.87, 99.74, 99.73, 99.70, 99.66 (C-1 $\left.{ }_{F}, 1_{E}, 1_{D}, 1_{C}, 1_{B}\right), 98.65\left(C-1_{A}\right), 81.71,81.40$, $81.35,81.34,81.32\left(\mathrm{C}-3_{\mathrm{F}}, 3_{\mathrm{E}}, 3_{\mathrm{D}}, 3_{\mathrm{C}}, 3_{\mathrm{B}}\right), 78.76\left(\mathrm{C}-3_{\mathrm{A}}\right), 57.58,56.62\left(\mathrm{OCH}_{3}-3_{\mathrm{F}}, 3_{\mathrm{E}}\right)$, $56.39\left(\mathrm{OCH}_{3}-3_{\mathrm{D}}, 3_{\mathrm{C}}, 3_{\mathrm{B}}\right), 56.36\left(\mathrm{OCH}_{3}-3_{\mathrm{A}}\right), 54.94\left(\mathrm{OCH}_{3}-1_{\mathrm{A}}\right), 21.04\left(\mathrm{CH}_{3} \mathrm{CO}\right)$; FDMS: $m / z 2212[\mathrm{M}]^{+}, 2235[\mathrm{M}+\mathrm{Na}]^{+}$. Anal. Calcd for $\mathrm{C}_{129} \mathrm{H}_{150} \mathrm{O}_{32}: \mathrm{C}, 70.03 ; \mathrm{H}, 6.83$. Found: C, 69.85; H, 6.75.

Methyl O-(2,6-di-O-benzyl-3-O-methyl- $\alpha$-D-mannopyranosyl)-l( $1 \rightarrow 4)-\mathrm{O}-(2,6-d i-\mathrm{O}-$ benzyl-3-O-methyl- $\alpha$-D-mannopyranosyl $)]_{4}-(1 \rightarrow 4)-2,6$-di-O-benzyl-3-O-methyl- $\alpha$-Dmannopyranoside (2). - A solution of $1(55 \mathrm{mg}, 24.9 \mu \mathrm{mol})$ in THF (1 mL) and $0.15 \mathrm{~N}$ $\mathrm{NaOMe}-\mathrm{MeOH}(1: 1,1.5 \mathrm{~mL})$ was stirred for $2 \mathrm{~h}$ at room temperature. Processing and chromatography on silica gel in 1:1 petroleum ether-EtOAc afforded 2 as an amorphous solid $(46.5 \mathrm{mg}, 86 \%) ;[\alpha]_{\mathrm{D}}+19.5^{\circ}\left(c 0.8, \mathrm{CHCl}_{3}\right) ;{ }^{1} \mathrm{H}$ NMR: $\delta 7.64-7.16(\mathrm{~m}, 60 \mathrm{H}$, aromatic H), 5.42-5.21 (m, $\left.5 \mathrm{H}, \mathrm{H}-1_{\mathrm{F}}, \mathrm{l}_{\mathrm{E}}, 1_{\mathrm{D}}, 1_{\mathrm{C}}, 1_{\mathrm{B}}\right), 4.79\left(\mathrm{~d}, 1 \mathrm{H}, J_{1.2} 1.7 \mathrm{~Hz}, \mathrm{H}-1_{\mathrm{A}}\right)$, 4.76-4.34 (m, $\left.24 \mathrm{H}, 12 \mathrm{PhCH}_{2}\right), 3.37\left(\mathrm{~s}, 3 \mathrm{H}, \mathrm{OCH}_{3} \mathrm{I}_{\mathrm{A}}\right), 3.25,3.20,3.19,3.18,3.17$, $3.10\left(6 \mathrm{~s}, 18 \mathrm{H}, \mathrm{OCH}_{3}-3_{\mathrm{A}}, 3_{\mathrm{B}}, 3_{\mathrm{C}}, 3_{\mathrm{D}}, 3_{\mathrm{E}}, 3_{\mathrm{F}}\right) ;{ }^{13} \mathrm{C}$ NMR: $\delta$ 138.7-138.3 (aromatic C-1), 128.4-127.3 (aromatic C), 99.86, 99.75, 99.74, 99.70, $99.68\left(\mathrm{C}-1_{\mathrm{F}}, 1_{\mathrm{E}}, 1_{\mathrm{D}}, 1_{\mathrm{C}}, 1_{\mathrm{B}}\right), 98.64$ $\left(\mathrm{C}-1_{\mathrm{A}}\right), 81.71,81.39,81.34,81.33,81.32\left(\mathrm{C}-3_{\mathrm{F}}, 3_{\mathrm{E}}, 3_{\mathrm{D}}, 3_{\mathrm{C}}, 3_{\mathrm{B}}\right), 78.76\left(\mathrm{C}-3_{\mathrm{A}}\right), 57.58$, $56.62\left(\mathrm{OCH}_{3}-3_{\mathrm{F}}, 3_{\mathrm{E}}\right), 56.40\left(\mathrm{OCH}_{3}-3_{\mathrm{D}}, 3_{\mathrm{C}}, 3_{\mathrm{B}}\right), 56.39\left(\mathrm{OCH}_{3}-3_{\mathrm{A}}\right), 54.95\left(\mathrm{OCH}_{3}-1_{\mathrm{A}}\right)$; FDMS: $m / z 2170[\mathrm{M}]^{+}$. Anal. Calcd for $\mathrm{C}_{127} \mathrm{H}_{148} \mathrm{O}_{31}: \mathrm{C}, 70.28 ; \mathrm{H}, 6.87$. Found: $\mathrm{C}$, $70.01 ; \mathrm{H}, 6.79$. 


\section{References}

[1] W.L. Smith and C.E. Ballou, J. Biol. Chem., 248 (1973) 7118-7125.

[2] D.E. Vauce, O. Mitsuhashi, and K. Bloch, J. Biol. Chem., 248 (1973) 2303-2309.

[3] Y.C. Lee and C.E. Ballou, J. Biol. Chem., 239 (1964) 3602-3603.

[4] G.R. Gray and C.E. Ballou, J. Biol. Chem., 246 (1971) 6835-6842.

[5] S.K. Maitra and C.E. Ballou, J. Biol. Chem., 252 (1977) 2459-2469.

[6] K.K. Yabusaki, R.E. Cohen, and C.E. Ballou, J. Biol. Chem., 254 (1979) 7282-7286.

[7] C.E. Ballou, Pure \& Appl. Chem., 53 (1981) 107-112.

[8] W. Liao, Y. Liu, and D. Lu, Carbohydr. Res., 260 (1994) 151-154.

[9] J.G. Buchanan and J.C.P. Schwarz, J. Chem. Soc., (1962) 4770-4777.

[10] M.E. Evans, Carbohydr. Res., 21 (1972) 473-475.

[11] D.A. Horne and A. Jordan. Tetrahedron Lett., (1978) 1357-1358.

[12] P.J. Garegg and H. Hultberg, Carbohydr. Res., 93 (1981) C10-C11.

[13] P.J. Garegg, H. Hultberg, and S. Wallin, Carbohydr, Res., 108 (1982) 97-101.

[14] R. Bose and R. Scheffold, Angew. Chem., 88 (1976) 578-579.

[15] T. Ogawa and S. Nakabayashi, Carbohydr. Res., 93 (1981) Cl-C5.

[16] R.R. Schmidt and J. Michel, Angew. Chem., Int. Ed. Engl., 19 (1980) 731-732.

[17] S. Sato, Y. Ito, T. Nukada, T. Nakahara, and T. Ogawa, Carbohydr. Res., 167 (1987) 197-210.

[18] S.S. Bhattacharjee and P.A.J. Gorin, Can. J. Chem., 47 (1969) I 195-1206.

[19] S. Xiong, F. Kong, and C. Yang, Youji Huaxue, 14 (1994) 280-285.

[20] M.A. Nashed, Carbohydr. Res., 60 (1978) 200-205.

[21] O.R. Martin, C.A.V. Hendricks, P.P. Deshpande, A.B. Cutler, S.A. Kane, and S.P. Rao, Carbohydr. Res. $196(1990) 41-58$. 